\title{
Role of Particulate Matter in Skin Inflammation
}

\section{Madeeha Arooj and Young-Sang Koh*}

Department of Microbiology and Immunology, School of Medicine and Brain Korea 21 PLUS Program, and Jeju Research Center for Natural Medicine, Jeju National University, Jeju, Korea

\author{
Corresponding \\ Young-Sang Koh \\ Department of Microbiology and Immunology, \\ Jeju National University School of Medicine, \\ 102 Jejudaehakno, Jeju 63243, South Korea \\ Phone : +82-64-754-3851 \\ Fax : +82-64-702-2687 \\ E-mail : yskoh7@jejunu.ac.kr
}

Received : February 28, 2019

Revised : March 16, 2019

Accepted : March 202019
No potential conflict of interest relevant to this article was reported.

Copyright (C) 2019 Journal of Bacteriology and Virology

CThis is an Open Access article distributed under the terms of the Creative Commons Attribution Non-Commercial License (http://creativecommons.org/ license/by-nc/3.0/).
Environmental pollution is a major global problem that affects human health. Among different types of environmental pollution, particulate matter (PM) has a key role. As PM spreads in the air so it causes different bronchial and skin inflammatory conditions. PM increases oxidative stress by ROS production and pro-inflammatory cytokines. As a result, matrix metalloproteinase is also produced, such as MMP1, MMP9 and MMP2 that is a known major cause of skin collagen degradation. Recently it has been shown that the PM worsens allergic conditions like dermatitis, psoriasis, and skin cancer. Different extensive efforts have been made across the globe to control this PM.

Key Words: Particulate matter, Pro-inflammatory cytokines

\section{INTRODUCTION}

World is facing environmental pollution from centuries and there are different types of pollution such as air, water and soil pollution (1). Nowadays air pollution has become one of the most alarming situations for the population due to its adverse effects on health and it must be taken seriously (2). In 2014, according to a survey of World Health Organization declared that air pollution is one of the main causes responsible for the premature deaths (3).

Due to increase in pollution, air is composed of different types of suspended particles, smoke from the vehicles, smog, poly aromatic hydrocarbons (PAHs) and biological contaminants including pollens, house mite dust allergens sulfates, nitrates and different carbon compounds collectively called as particulate matter (PM) (2). PM is one of the biggest causes of air pollution and is defines as fine particles that can be inhales easily and cause different respiratory and skin inflammatory problems like asthma, shortening of breath, lungs cancer and dermatitis, psoriasis, etc. (3). It has been reported that PM increases the oxidative stress and inflammatory cytokines that worsens the preexisting allergies and conditions (1). This had been further explored and showed that PM is classified into different categories depending upon diameter and origin of source (4). Due to its adverse effects on health, World Health Organization (WHO) suggested a limited concentration of PM10 should not exceed from $20 \mu \mathrm{g} / \mathrm{m}^{3}$ per day annually (5). In this review, we introduce recent advances in PM discovery and effects on skin inflammation. 


\section{Classification of PM}

Depending upon the size and diameter PM is classified into three different categories: i.e. particulate matter, fine particulate matter and ultra-fine particles (UFP) $(1,4)$. PM whose size is less than $10 \mu \mathrm{m}$ called as PM10 and mostly inhalable (6). Infiltrations of PM10 cause different pulmonary diseases like asthma and lungs cancer $(1,7)$. Fine particulate matter was discovered in 1990 and size is about $2.5 \mu \mathrm{m}$ (4). Nowadays, fine PM is increasing tremendously and composed of different nitrates, sulfates and organic black carbon compounds (1). Except respiratory and skin diseases PM2.5 also associated with exacerbation of different heart diseases.

Recently third category of PM, known as ultra-fine particles (UFP) size less than $100 \mathrm{~nm}$ is discovered (1). According to WHO, UFP is also a carcinogen. Main sources of UFP includes both indoor and outdoor, i.e., fumes from cooking, smoke from engines, industrial and diesel exhaust particle $(7,8)$. Among all PM, UFP has worse effects on human health; it directly penetrates into the blood stream and accumulates into the lungs results in chronic obstructive pulmonary disease (COPD) (1).

\section{PM associated with skin inflammatory conditions}

Skin is the largest organ which covers whole body and is called as first line of defense $(1,6)$. Skin is composed of three different layers epidermis (the outer most layers), dermis and hypodermis $(9,10)$. The stratum corneum derived from Latin word "horny layer" and is outer most layer of the epidermis consists of dead cells and continuously sheds keratinocytes (10). Skin is also covered with normal microbiota provides a protective barrier against different pathogens and stimuli. When skin gets exposed to different stimuli like toxins, PM and contaminants normal microflora gets disturbed cause toxicity in skin and cause different skin inflammatory conditions like atopic dermatitis (AD), psoriasis, acne, skin aging and skin cancer (10). Some recent studies conducted and suggested that particulate matter worsens the pre-existing skin allergic conditions like dermatitis, psoriasis and acne. Previous studies only suggested that PM do not directly cause any skin inflammatory condition unless a preexisting condition. Atopic dermatitis is a chronic and inflammatory skin condition in which patient suffers from itching and eczema (8). According to different epidemiological investigations, it is suggested that AD patients when exposed to outdoor and indoor pollutants it worsens the preexisting allergic conditions because it impairs the epidermal barrier function and water loss from the skin (9). Recently a survey is conducted and suggested that along with PM, weather conditions play a critical role in exacerbating the dermatitis (9). Acne is another skin inflammatory condition which affects sebaceous gland and results in skin changes and is characterized by pustules, papules and nodules $(1,10)$. Polycyclic aromatic hydrocarbons (PAHs) including cigarette smoke plays an important role in acne formation because it increases the secretion of pro-inflammatory cytokine like interleukin (IL)-1 $\alpha$. This pro-inflammatory cytokine is present in the pustules of acne. Different studies conducted and suggested that acne symptoms worsen by cigarette smoke by increasing ROS and IL-8 production (1). Psoriasis is also a paradigmatic chronic inflammatory skin condition and etiology is not known yet. Chronic relapsing dermatitis is also called as psoriasis. So, the role of T cell immunity in PM induced psoriasis is not yet known.

\section{Disruption of skin barrier}

The general mechanism through which particulate matter, cigarette smoke and diesel exhaust induce different inflammatory skin conditions is described below. PM and all these contaminants composed of PAHs. When skin exposed to PM impairment in the epidermis occurs due to activation of signaling cascade. First one is ROS production and second is aryl hydrocarbon receptor (AhR) activation, which is also called as a receptor for PAHs in human keratinocytes $(1,3)$. Upon cellular entry of any type of PAHs binds to its known receptor AhR in the cytoplasm of keratinocytes (1). Receptor ligand interaction takes place between PAHs and AhR and helps in transcription by activating different transcriptional factors (1). Increased production of ROS induces production of pro-inflammatory cytokines, i.e., TNF- $\alpha$ and IL-8 from keratinocytes and 
MAPK signaling pathway including ERK1/2, JNK and p38 gets activated. MAPK pathway then induces activation of different transcriptional factors, i.e., NF- $\mathrm{KB}$ and AP-1.

Due to all combined actions, ROS production, enhancement in pro-inflammatory cytokines, MAPK activation matrix metallo proteinase abbreviated as MMPs also called as matrixins gets activated. MMPs play an important role in cell behavior, apoptosis and cells degeneration. Collagens in the skin is degraded by MMPs and results in impairment of skin barrier, water loss that is a major cause of all skin inflammatory conditions, i.e., skin aging, skin cancer, alopecia and different skin diseases.

\section{In vivo study}

Authors also conducted in vivo study to investigate the role of AhR whether it has a role in skin cancer. For this purpose, $\mathrm{AhR}^{+/+}$and deficient mice are used. Air borne particulate extracts was used as stimuli. AhR deficient mice do not show any carcinogenic effect on skin while $\mathrm{AhR}^{+/+}$has showed squamous cell carcinoma because it express CYP1A1 expression which is a transcription factor. This in vivo study suggested that AhR plays a major role in carcinogenesis (1).

\section{Closing Remarks}

A new gateway through which we get to know about the particulate matter and its effects on health. Here we have summarized that particulate matter has very alarming effects on human health especially on skin and cause different skin inflammatory conditions. Further investigations show that PM induces ROS production which in turn increases production of pro-inflammatory cytokines that is a major cause of skin cancer and different skin inflammatory conditions

\section{Acknowledgements}

This research was supported by the 2019 Scientific Promotion Program funded by Jeju National University

\section{REFERENCES}

1) Kim EK, Cho D, Park HJ. Air pollution and skin diseases: Adverse effects of airborne particulate matter on various skin diseases. Life Sci 2016;152:126-34.

2) Franck $U$, Odeh $S$, Wiedensohler A, Wehner B, Herbarth $O$. The effect of particule size on cardiovascular disorders - the smaller the worse. Sci Total Environ 2011:409:4217-21.

3) Li N, Hao M, Phalen RF, Hinds WC, Nel AE. Particulate air pollutants and asthma. A paradigm for the role of oxidative stress in PM-induced adverse health effects. Clin Immunol 2003;109:250-65.

4) Li N, Sioutas C, Cho A, Schmitz D, Misra C, Sempf J, et al. Ultrafine particulate pollutants induce oxidative stress and mitochondrial damage. Enivron Health Perspect 2003;111:455-60.

5) Kappos AD, Bruckman $P$, Eikmann $T$, Englert $N$, Heinrich U, Hoppe $P$, et al. Health effects of particles in ambient air. Int J Hyg Enivron Health 2004;207:399-407.

6) Kim J, Kim EH, Oh I, Jung K, Han Y, Cheong HK, et al. Symptoms of atopic dermatitis are influenced by outdoor air 
pollution. J Allergy Clin Immunol 2013;132:495-8.

7) Pappu R, Carrozzi VR, Ota N, Ouyang W, Hu Y. The IL-17 family cytokines in immunity and disease. J Clin Immunol 2010;30:185-95.

8) Jin PS, Li Z, Choi EK, Lee S, Kim YK, Seo EY, et al. Urban particulate matter in air pollution penetrates into the barrier-disrupted skin and produces ROS-dependent cutaneous inflammatory response in vivo. J Dermatol Sci 2018;91:175-83.

9) Kim YM, Kim J, Jung K, Eo S, Ahn K. The effects of particulate matter on atopic dermatitis symptoms are influenced by weather type: Application of spatial synoptic classification (SSC). Int J Hyg Enivron Health 2018;221:823-9.

10) Pan TL, Wang PW, Aljuffali IA, Huang CT, Lee CW, Fang JY. The impact of urban particulate pollution on skin barrier function and the subsequent drug absorption. J Dermatol Sci 2015;78:51-60. 